\title{
Dendrobium mixture regulates hepatic gluconeogenesis in diabetic rats via the phosphoinositide-3-kinase/protein kinase B signaling pathway
}

\author{
XINJUN LIN ${ }^{1}$, HONG SHI $^{1}$, YI CUI ${ }^{2}$, XIAONING WANG $^{1}$, JIEPING ZHANG $^{1}$, WENZHEN YU ${ }^{1}$ and MIN WEI ${ }^{1}$ \\ ${ }^{1}$ Institute of Integrated Chinese and Western Medicine, Fujian University of Traditional Chinese Medicine, Fuzhou, \\ Fujian 350122; ${ }^{2}$ Department of Ophthalmology, Fujian Medical University Union Hospital, Fuzhou, Fujian 350001, P.R. China
}

Received June 6, 2017; Accepted January 22, 2018

DOI: $10.3892 /$ etm.2018.6194

\begin{abstract}
The present study aimed to evaluate the impact of dendrobium mixture (DMix) on the gene and protein expression of insulin signaling pathway-associated factors in the livers of diabetic rats. The molecular mechanisms by which DMix inhibits gluconeogenesis were also investigated. A total of 47 female Wistar rats were used in the present study. Of these, 11 rats were randomly selected as healthy controls and diabetes was induced in the remaining 36 rats by administering a high-fat and high-sugar diet for 6 weeks, followed by two intraperitoneal injections of streptozotocin. The 36 rats were screened for diabetes and then randomly divided into three groups: Model, metformin and DMix groups. Following 12 weeks of treatment, the fasting blood glucose (FBG), glycosylated serum protein (GSP), serum insulin, blood lipids [total cholesterol (Tch) and triglycerides (TG)], alanine transaminase (ALT) and aspartate transaminase (AST) were assessed. In addition, hematoxylin and eosin staining was used for histomorphological examination of the liver tissues. The mRNA expression of insulin receptor (InsR), forkhead box protein O1 (FoxO1), phosphoenolpyruvate carboxykinase (PEPCK) and glucose 6-phosphatase (G6Pase) in the liver was measured with reverse transcription-quantitative polymerase chain reaction and the protein expression of InsR, phosphoinositide-3-kinase (PI3K), phosphorylated (p)-PI3K, protein kinase B (Akt), p-Akt, FoxO1, PEPCK and G6Pase in the liver was measured by western blot analysis. The FBG, GSP, InsR, Tch, TG, ALT and AST levels were significantly lower in the DMix-treated group compared with the model group $(\mathrm{P}<0.05)$. In addition,
\end{abstract}

Correspondence to: Professor Hong Shi, Institute of Integrated Chinese and Western Medicine, Fujian University of Traditional Chinese Medicine, 1 Qiuyang Road, Fuzhou, Fujian 350122, P.R. China

E-mail: shihong3327@sina.com

Key words: dendrobium mixture, insulin resistance, phosphoinositide-3-kinase/protein kinase B signaling pathway, gluconeogenesis
DMix treatment notably improved liver histopathology and significantly increased the gene and protein expression of InsR, PI3K and Akt $(\mathrm{P}<0.05)$. DMix treatment also significantly reduced the gene and protein expression of FoxO1, PEPCK and G6Pase $(\mathrm{P}<0.05)$. DMix effectively reduced FBG and blood lipids and significantly improved liver function and insulin resistance in diabetic rats, possibly by regulating the gene and protein expression of molecules associated with the PI3K/Akt signaling pathway.

\section{Introduction}

Diabetes is a serious public health concern worldwide. In China, the number of adults with diabetes or pre-diabetes (age $\geq 20$ ) was 114 and 493 million in 2010, respectively (1); type II diabetes accounted for $90 \%$ of these cases (2). Absolute insulin deficiency, or insulin resistance may lead to elevated blood glucose levels in patients with diabetes (3). The phosphoinositide-3-kinase/protein kinase B (PI3K/Akt) signaling pathway is critical to insulin function (4). Previous studies have revealed that any dysregulation in the expression, structure and function of proteins in the PI3K/Akt signaling pathway disrupts insulin signal transduction and weakens the physiological effects of insulin, which leads to insulin resistance and metabolic disorders $(5,6)$. Gluconeogenesis is the process by which non-sugar precursors, including lactic acid, glycerol and raw sugar amino acids, are converted into glucose (7). Half of all glucose consumed by the body and used as energy for vital organs is produced via gluconeogenesis (8).

Gluconeogenesis primarily occurs in the liver, and hepatic gluconeogenesis determines fasting blood glucose (FBG) levels and serves a key role in the conservation of sugar (9). Phosphoenolpyruvate carboxykinase (PEPCK) and glucose 6-phosphatase (G6Pase) are important rate-limiting enzymes that regulate hepatic gluconeogenesis (10). Forkhead box protein $\mathrm{O} 1$ (FoxO1) is a transcription factor that is negatively regulated by insulin signaling and is important for the regulation of liver metabolism (11). Liver hormones regulate FoxO1 activity to modulate PEPCK and G6Pase gene expression (12).

The drugs commonly used to treat type 2 diabetes are insulin, biguanide and sulfonylureas (13). As the disease progresses, the prolonged administration of these drugs may 
cause side effects, including metabolic imbalance, gastrointestinal systemic damage, kidney and liver damage and tumorigenesis (13). Therefore, there is an urgent need for novel agents that improve the symptoms of diabetes and stabilize blood sugar. Several previous studies have demonstrated that numerous traditional Chinese medicines (TCMs), including Si-haung Hypoglycemic Granules and Rhizoma Coptidis apozem, promote glucose and lipid metabolism by regulating the insulin signal transduction pathway $(14,15)$. Previous studies have demonstrated that dendrobium mixture (DMix) significantly alleviates the clinical symptoms of diabetes, corrects impaired glucose tolerance and improves insulin resistance $(16,17)$. In addition, all patients with diabetes who were treated with DMix took significantly fewer western medications at a 5-year follow-up, which suggests that this treatment may have a better efficacy than metformin, which is the most common hyperglycemic agent of biguanide $(16,17)$.

In the present study, a rat model of type II diabetes was established using a high-fat and high-sugar diet with intraperitoneal injections of streptozotocin (STZ). The rat model was used to investigate the molecular mechanisms by which DMix regulates the insulin signaling pathway to inhibit gluconeogenesis and improve sugar conservation.

\section{Materials and methods}

Research subjects. A total of 47 Specific-pathogen-free (SPF) healthy female Wistar rats (age, 2.5 months) weighing $200 \pm 20 \mathrm{~g}$ were purchased from Shanghai Laboratory Animal Center (Shanghai, China). The animals were housed at the Experimental Animal Center at the Fujian University of TCM (Fuzhou, China) in an SPF grade laboratory at room temperature $\left(25^{\circ} \mathrm{C}\right)$ with $80 \%$ relative humidity. A total of five rats were housed per cage in a 12-h light/dark cycle with free access to food and water. The basic animal feed and the high-fat feed were purchased from the Experimental Animal Center at the Fujian University of TCM. Based on a previous study (12), the high-fat feed comprised $60.7 \%$ basic feed, $10 \%$ lard, $15 \%$ sucrose, $10 \%$ egg yolk powder, $4 \%$ cholesterol and $0.3 \%$ cholate.

Experimental reagents. DMix (15 g dendrobium, $20 \mathrm{~g}$ astragalus, $8 \mathrm{~g}$ schisandra, $15 \mathrm{~g}$ pueraria, $15 \mathrm{~g}$ salvia, $15 \mathrm{~g}$ rehmannia and $8 \mathrm{~g}$ earthworms) was purchased from Guoyitang Clinic, Fujian University of TCM. Metformin (glucophage) tablets were purchased from the Sino-American Shanghai Squibb Pharmaceutical Co., Ltd. (Shanghai, China), with a production specification of $0.85 \mathrm{~g} /$ tablet (national medicine approval number H20023370). The other experimental reagents included an insulin ELISA detection kit (cat no. F6403; Westang Biotechnology Co., Ltd., Shanghai, China), a blood glucose meter and blood glucose strips (Yuyue Medical Equipment \& Supply Co., Ltd. (Nanjing, China), TRIzol reagent (Invitrogen; Thermo Fisher Scientific, Inc., Waltham, MA, USA), anhydrous ethanol and isopropyl alcohol. Agarose was purchased from Sigma-Aldrich (Merck, KGaA, Darmstadt, Germany). The following antibodies were used: Insulin receptor (InsR, cat. no. 3025), Akt (cat. no. 4685) and phosphorylated (p)-Akt (cat. no. 9611) (all Cell Signaling Technology, Inc., Danvers, MA, USA), anti- $\beta$-actin (cat. no. SAB1305567; Sigma-Aldrich;
Merck KGaA), PI3K, p-PI3K, FoxO1, PEPCK and G6pase (all Abcam, Cambridge, MA, USA). A SYBR-Green I quantitative PCR kit (Takara Bio, Inc., Otsu, Japan) was purchased from Thermo Fisher Scientific, Inc. and PCR primers were produced by Invitrogen (Thermo Fisher Scientific, Inc.).

Grouping and processing. A total of 47 female Wistar rats were used. Of these, 11 rats were fed a basic diet and made up the healthy control group (CTR group). The remaining rats were fed a high-fat/high-sugar diet for 6 weeks, followed by two intraperitoneal injections of streptozotocin (STZ, $25 \mathrm{mg} / \mathrm{kg}$ ) once per day. Rats with FBG levels $>7.0 \mathrm{mmol} / 1$ or random blood glucose levels $>16.7 \mathrm{mmol} / 1$ on 2 consecutive days were selected as the diabetic models. Following stratification by body weight and blood sugar, the 36 diabetic rats were randomly divided into three groups of 12 as follows: The model group, the DMix group and the metformin group (positive control). The present study was approved by the Ethics Committee of Fujian University of TCM.

The healthy control rats were fed a basic diet and received normal saline by gavage. The model group of diabetic rats were fed a basic diet and received normal saline by gavage. The DMix group of diabetic rats was fed a basic diet and received DMix (17.2 $\mathrm{g} / \mathrm{kg} /$ day) by gavage. The metformin group of diabetic rats was fed a basic diet and received metformin $(100 \mathrm{mg} / \mathrm{kg} /$ day) by gavage. Following 12 weeks of treatment all animals were fasted without water for 1 night and all rats were subsequently weighed at 8.00 am and then anaesthetized using intraperitoneal $10 \%$ urethane $(1,000 \mathrm{mg} / \mathrm{kg})$. Rapid laparotomy was performed prior to sample collection. Blood was collected from the abdominal aorta, centrifuged at $1,625 \mathrm{x} \mathrm{g}$ for $20 \mathrm{~min}$ at room temperature to collect serum and preserved at $-20^{\circ} \mathrm{C}$ for later experiments. The livers from all rats were collected, dissected and divided. Liver tissue (100 mg) was placed into $1.5 \mathrm{ml}$ Eppendorf tubes, snap frozen in liquid nitrogen and stored at $-80^{\circ} \mathrm{C}$ for reverse transcription-quantitative polymerase chain reaction (RT-qPCR) and western blot analysis. The remaining tissue was fixed in $4 \%$ paraformaldehyde at $4^{\circ} \mathrm{C}$ for $72 \mathrm{~h}$. for histopathological examination using hematoxylin and eosin (H\&E) staining.

Hematoxylin \& eosin $(H \& E)$ staining. H\&E histology was performed to examine the histopathological changes in the liver. Liver tissues were dissected and fixed in $4 \%$ paraformaldehyde at $4^{\circ} \mathrm{C}$ for $72 \mathrm{~h}$, dehydrated and embedded in paraffin blocks. Liver sections ( $3 \mathrm{~mm}$ ) were sliced backward from the optic chiasma. Sections were deparaffinized and hydrated with decreasing concentrations of alcohol, stained with H\&E (Stained with Hematoxylin for $5 \mathrm{~min}$ at room temperature and $0.5 \%$ eosin for $1 \mathrm{~min}$ at room temperature), and observed using a light microscope (DFC310 FX; Leica, Wetzlar, Germany) at magnification, $\mathrm{x} 200$.

Measurements. Blood glucose was measured using the glucose oxidase method according to the protocol of a previous study (18). Serum triglycerides (TG), total cholesterol (Tch), low-density lipoprotein-cholesterol (LDL-C), alanine transaminase (ALT), aspartate transaminase (AST) and glycosylated serum protein (GSP) levels were measured using 
an automated LX-20 Pro biochemistry analyzer (Beckman Coulter, Inc., Brea, CA, USA). Insulin ELISA kits were used to measure fasting insulin (FIN) levels and the homeostasis model assessment of insulin resistance (HOMA-IR) was used to quantify glycosylated hemoglobin. HOMA-IR, which indicates the degree of insulin resistance, was calculated according to the following equation: HOMA-IR=(FBG $\times$ FIN $) / 22.5$.

$R T-q P C R$. RT-qPCR was used to measure InsR, FoxO1, PEPCK and G6Pase gene expression. A total of $100 \mathrm{mg}$ of frozen liver tissue was homogenized to extract total RNA using TRIzol ${ }^{\circledR}$ (Thermo Fisher Scientific, Inc.). RNA integrity was tested by electrophoresing the RNA in a denaturing formaldehyde gel. Total RNA was then reverse transcribed into cDNA using a PrimeScriptTM II 1st Strand cDNA Synthesis kit (Takara Bio, Inc.). The following primers were used for RT-qPCR: InsR forward, 5'-TTTTTGTCCCCAGGCCATCC-3' and reverse, 5'-CCTGTGCTCCTCCTGACTTG-3'; Foxo1 forward, 5'-CCCAGGCCGGAGTTTAACCA-3' and reverse, 5'-AGC AGGCTCAGGTTGCTCAT-3'; PEPCK forward, 5'-GGA AGCGGATACGGTGGGAA-3' and reverse, 5'-GGAAGG CTGCTGCCAGGTAT-3'; G6Pase forward, 5'-GCTCCT GGGACAGACACACA-3' and reverse, 5'-CCACACTGGGTT GCACAAGG-3'; and GAPDH forward, 5'-GTTACCAGG GCTGCCTTCTC-3' and reverse, 5'-GGGTTTCCCGTTGAT GACC-3' as the reference gene. Probe primers were designed from the conserved sequences of target genes obtained from Invitrogen (Thermo Fisher Scientific, Inc.), and were used to generate PCR products with a SYBR Green I quantitative PCR kit (Takara Bio Inc.) and amplify target genes. An ABI7500 Real-Time PCR system (Applied Biosystems; Thermo Fisher Scientific, Inc.) was used to analyze copy numbers with the following thermocycling conditions: Denaturation, $95^{\circ} \mathrm{C}$ for $10 \mathrm{sec} ; 30$ cycles at $95^{\circ} \mathrm{C}$ for $10 \mathrm{sec} ; 60^{\circ} \mathrm{C}$ for $20 \mathrm{sec} ; 95^{\circ} \mathrm{C}$ for $60 \mathrm{sec} ; 55^{\circ} \mathrm{C}$ for $30 \mathrm{sec}$; and $95^{\circ} \mathrm{C}$ for $30 \mathrm{sec}$. The results were determined using the $2^{-\Delta \Delta C q}$ method (19).

Western blot analysis. Western blotting was used to determine the total protein expression of InsR, PI3K, Akt, FoxO1, PEPCK and G6Pase, as well as the phosphorylation of PI3K and Akt. A total of $100 \mu \mathrm{g}$ purified protein extract was separated by $12 \%$ SDS-PAGE gel and transferred to nitrocellulose membranes. Non-specific proteins were blocked with bovine serum albumin (Beijing Solarbio Science \& Technology Co., Ltd., Beijing, China) for $1 \mathrm{~h}$ at room temperature and the membranes were incubated with primary antibodies $(1: 1,000)$ overnight at $4^{\circ} \mathrm{C}$. The membranes were washed with TBS-Tween solution and subsequently incubated for $1 \mathrm{~h}$ at room temperature with horseradish peroxidase (HRP)-labeled secondary antibodies (1:500, anti-rabbit IgG, cat. no. ZB-2301; anti-mouse IgG, cat. no. ZB-2305; each, OriGene Technologies, Inc., Rockville, MD, USA). The membranes were then washed twice. $\beta$-actin was used as the internal control. The band intensity was developed using an enhanced chemiluminescence-Plus kit (cat. no. RPN2132; GE Healthcare Life Sciences, Little Chalfont, UK). Images were taken with a Bio-Image Analysis system (Bio-Rad Laboratories, Inc., Hercules, CA, USA) and the bands were analyzed using ImageJ software (1.48 u) National Institutes of Health, Bethesda, MD, USA).

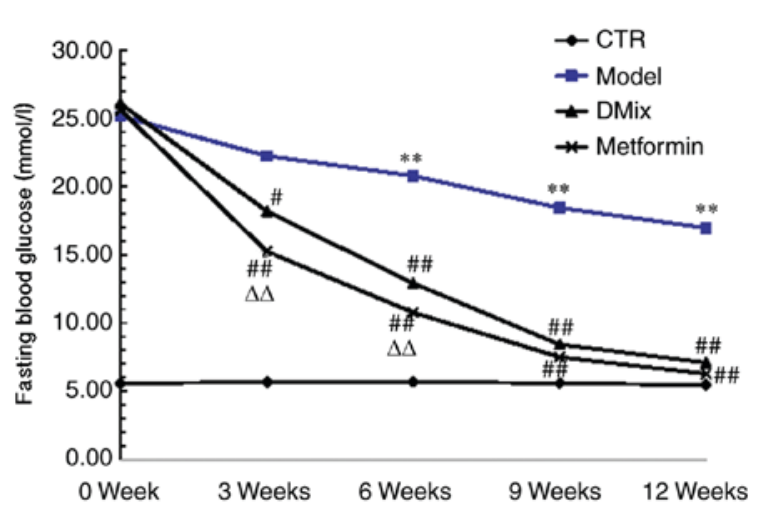

Figure 1. Fasting blood glucose levels in the different groups at 0,3,6,9 and 12 weeks. ${ }^{* *} \mathrm{P}<0.01$ vs. CTR group; ${ }^{\#} \mathrm{P}<0.05$ and ${ }^{\# \#} \mathrm{P}<0.01$ vs. Model group; ${ }^{\Delta \Delta} \mathrm{P}<0.01$ vs. DMix group. CTR, control.

Statistical analysis. The experimental data was analyzed using SPSS 19.0 (SPSS, Inc., Chicago, IL, USA) and are presented as the mean \pm standard deviation. All experiments were performed in triplicate. One-way analysis of variance was used to compare the mean values of samples across multiple groups. An LSD post-hoc test and Dunnett's T3 post-hoc test were also subsequently performed. $\mathrm{P}<0.05$ was considered to indicate a statistically significant result.

\section{Results}

General condition of animals during the construction of the rat model. The healthy control rats were active with clean, well-groomed hair. They exhibited no significant changes in urination and bowel movement frequency compared with the diabetic model groups (data not shown). The diabetic model rats were observed to be less active, with duller hair. Their intake of food and water increased significantly compared with the control rats, as did their frequency of urination and bowel movements (data not shown). No diarrhea or deaths occurred across all groups.

Changes in serum glucose and lipid metabolism FBG. Following 6 weeks of a high-fat/high-sugar diet and two intraperitoneal injections of STZ, the blood glucose levels of all diabetic rats were $>25 \mathrm{mmol} / \mathrm{l}$. There were no significant differences in blood glucose level among the different diabetic groups prior to treatment (data not shown). However, the FBG levels in the model group diabetic rats were significantly higher compared with the healthy control rats at 6,9 and 12 weeks $(\mathrm{P}<0.01$; Fig. 1). Following the administration of DMix and metformin, the FBG levels of the rats were significantly reduced at 3 ( $\mathrm{P}<0.05$ for DMix; $\mathrm{P}<0.01$ for metformin), 6, 9 and 12 weeks (all $\mathrm{P}<0.01$ ) compared with the diabetic model group. The FBG levels were significantly lower in the metformin group at 3 and 6 weeks compared with the DMix group $(\mathrm{P}<0.01)$, but no significant differences were observed between the two groups at 9 and 12 weeks.

GSP, FIN and HOMA-IR. Following 12 weeks of treatment, GSP, FIN and HOMA-IR were significantly elevated in the diabetic model group compared with the control group $(\mathrm{P}<0.01$; Fig. 2). However, GSP, FIN and HOMA-IR were significantly 

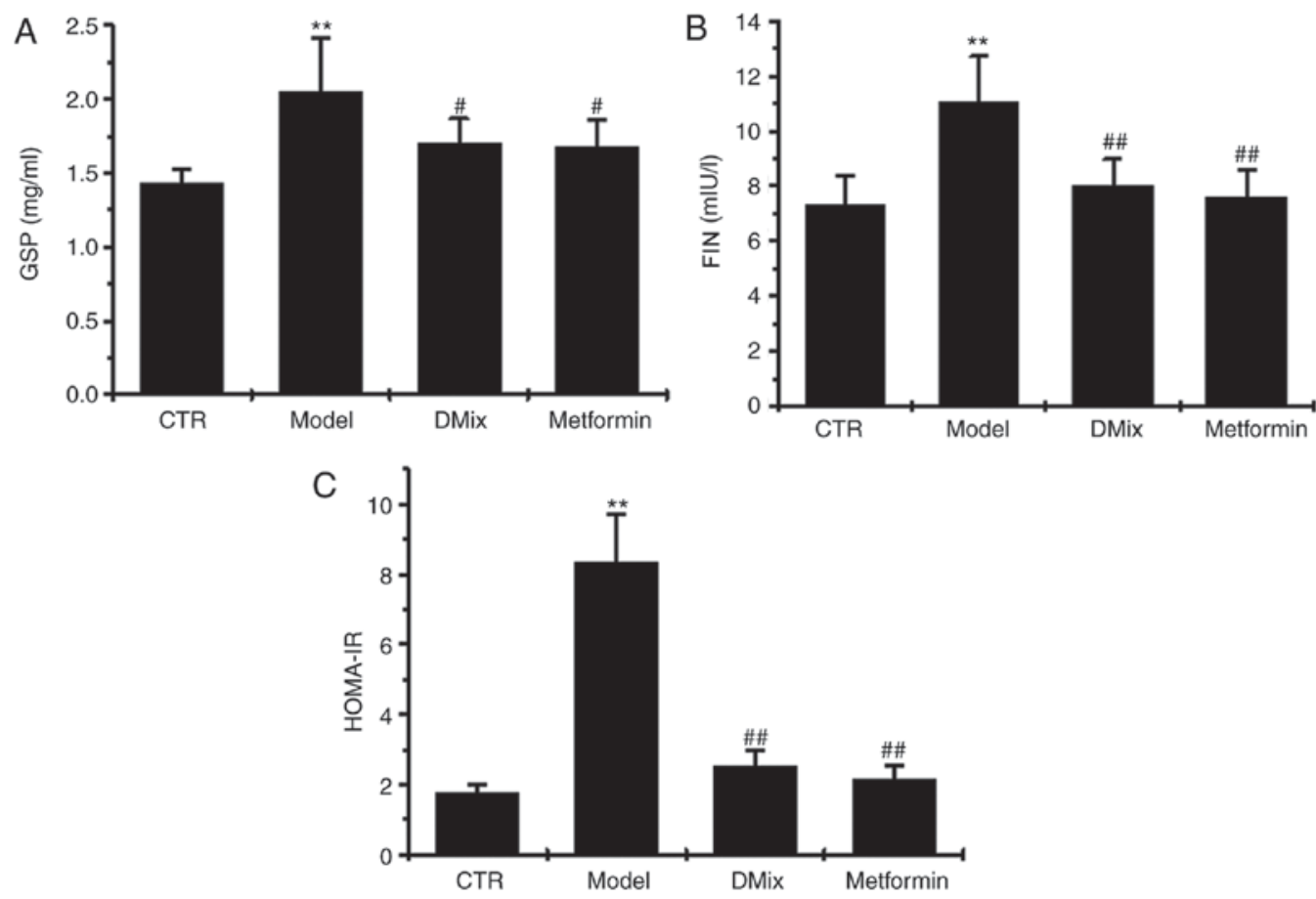

Figure 2. Serum glucose and mRNA levels were measured at 12 weeks following treatment. (A) GSP levels were measured with an automated LX-20 Pro biochemistry analyzer. (B) ELISA assays were performed to measure the FIN levels. (C) HOMA-IR was calculated according to the following equation: HOMA-IR=(fasting blood glucose $\mathrm{x}$ FIN)/22.5. ${ }^{* *} \mathrm{P}<0.01$ vs. CTR group; ${ }^{\#} \mathrm{P}<0.05$ and ${ }^{\# \#} \mathrm{P}<0.01$ vs. Model group. GSP, glycosylated serum protein; FIN, fasting insulin; HOMO-IR, homeostasis model assessment of insulin resistance; CTR, control.

lower in the DMix and metformin groups compared with the diabetic model group ( $\mathrm{P}<0.05$ for GSP; $\mathrm{P}<0.01$ for FIN and HOMA-IR).

Tch, TG, LDL, ALT and AST. Following 12 weeks of treatment the Tch, TG and LDL levels were significantly elevated in the diabetic model group compared with the control group $(\mathrm{P}<0.01$; Fig. 3A). However, the Tch, TG and LDL levels were significantly lower in the DMix group compared with the diabetic model group $(\mathrm{P}<0.05$ for Tch and LDL; $\mathrm{P}<0.01$ for TG), and were very similar to that of the control group $(\mathrm{P}>0.05)$. In the metformin group, the Tch and LDL levels were significantly lower compared with the diabetic model group $(\mathrm{P}<0.01)$, and were very similiar to the healthy controls (P>0.05). The ALT and AST levels were significantly elevated in the diabetic model group compared with the healthy control group $(\mathrm{P}<0.01$; Fig. 3B). The levels of ALT and AST were significantly lower in the DMix $(\mathrm{P}<0.01)$ and metformin $(\mathrm{P}<0.05$ for ALT; $\mathrm{P}<0.01$ for AST) groups compared with the diabetic model group. ALT levels were significantly higher in the DMix and the metformin group compared with the healthy control group $(\mathrm{P}<0.01)$. The AST levels in the DMix group were also very similar to the healthy controls $(\mathrm{P}>0.05)$. However, the metformin group demonstrated significantly higher levels compared with the healthy control group $(\mathrm{P}<0.01)$.

Liver histopathology. The H\&E stained liver tissues were examined by light microscopy (Fig. 4). This revealed that the majority of the rat livers from the healthy control group had a clear lobular structure and evenly sized hepatocytes radially distributed from the center of the central veins (Fig. 4A). In all of the healthy livers, the hepatic cord was arranged neatly.
The small arteries, veins and bile ducts had a normal structure in the portal area and the sinusoids were clearly visible. In all rat livers form the diabetic model group, the hepatocytes exhibited an altered shape with unclear cell contours (Fig. 4B). In these livers, the cord-like arrangement of the liver lobule had disappeared and the connections between hepatocytes were loose. In addition, diabetic model rats had an enlarged hepatic sinus, notable inflammatory cell exudation, connective tissue hyperplasia and an abnormal structure of the central veins and portal area. The rat livers from the DMix group had neatly arranged hepatocytes and the structure of the hepatic cord and the hepatic sinuses was improved compared with the untreated diabetic rats (Fig. 4C). The DMix rats exhibited no clear inflammatory cell infiltrations and collagen connective tissue hyperplasia was rare. The diabetic rats treated with metformin had a crooked hepatic cord structure, expanded hepatic sinuses and connective tissue hyperplasia in part of the portal area (Fig. 4D).

Effect of DMix on hepatic InsR, FoxO1, PEPCK and G6Pase $m R N A$ expression. The mRNA expression of InsR was significantly lower in the diabetic model group compared with the healthy control group $(\mathrm{P}<0.01)$, whereas FoxO1 $(\mathrm{P}<0.01)$, PEPCK $(\mathrm{P}<0.05)$ and G6Pase $(\mathrm{P}<0.01)$ mRNA expression was significantly higher compared with the control group (Fig. 5). InsR mRNA expression was significantly higher in the DMix and metformin groups compared with the diabetic model group $(\mathrm{P}<0.05)$, while FoxO1 $(\mathrm{P}<0.05)$, PEPCK $(\mathrm{P}<0.05)$ and G6Pase $(\mathrm{P}<0.01$ for DMix; $\mathrm{P}<0.05$ for metformin) mRNA expression was significantly lower in the DMix group compared with the diabetic model group. The DMix and metformin mRNA levels were similar to those of the healthy controls. 


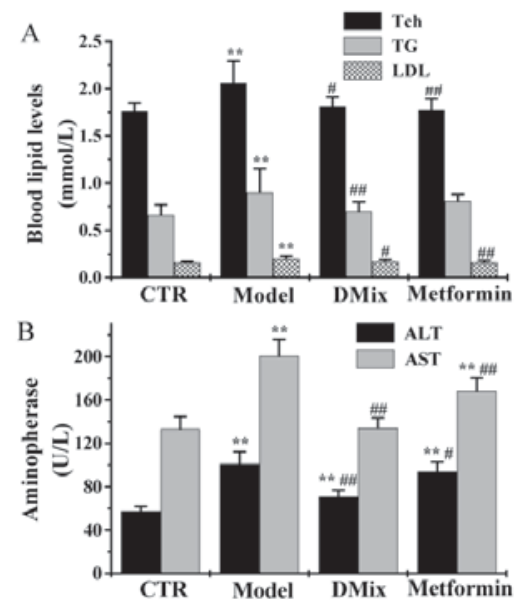

Figure 3. Blood lipid, ALT and AST levels at 12 weeks following treatment. (A) Blood lipid levels were measured in each group. (B) ALT and AST levels were measured with an automated LX-20 Pro biochemistry analyzer. ${ }^{* *} \mathrm{P}<0.01$ vs. CTR group; ${ }^{\#} \mathrm{P}<0.05$ and ${ }^{\# \#} \mathrm{P}<0.01$ vs. Model group. ALT, alanine transaminase; AST, aspartate transaminase; Tch, total cholesterol; TG, triglycerides; CTR, control; LDL, low-density lipoprotein.
A
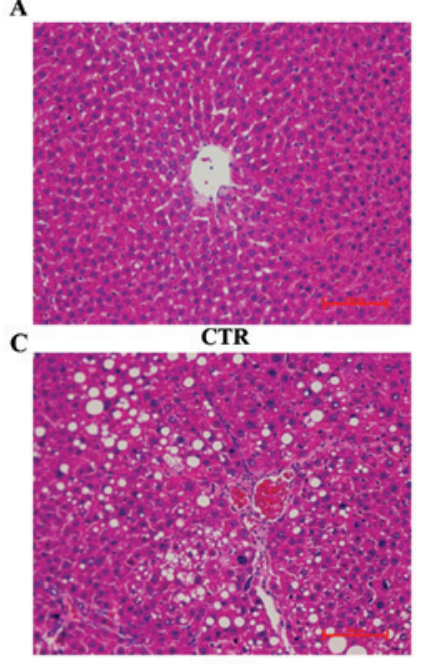

DMix
B
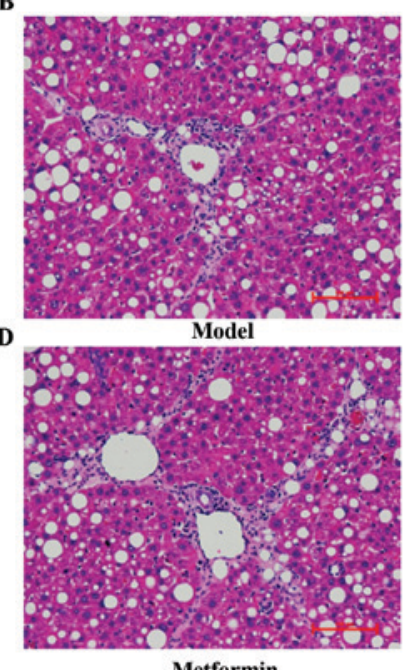

Metformin
Figure 4. Histopathological changes observed in hematoxylin and eosin stained liver tissues by light microscopy at magnification, x200. (A) CTR, (B) Model, (C) DMix and (D) Metformin group. Scale bar, $100 \mu \mathrm{m}$. Representative images are presented. CTR, control; DMix, dendrobium mixture.

Effect of DMix on hepatic InsR, PI3K, p-PI3K, Akt, p-Akt, FoxO1, PEPCK and G6Pase protein expression. There were no notable differences in the PI3K and Akt expression levels in the diabetic model group compared with the healthy control group (Fig. 6A). However, p-PI3K/PI3K, p-Akt/Akt and InsR expression levels were significantly lower in the diabetic model group compared with the healthy control group ( $\mathrm{P}<0.05$; Fig. 6B-D). The FoxO1, PEPCK and G6Pase expression levels were significantly higher in the diabetic model group compared with the healthy control group $(\mathrm{P}<0.05$; Fig. 6C and D). The levels of p-PI3K/PI3K $(\mathrm{P}<0.01)$, p-Akt/Akt $(\mathrm{P}<0.05)$ and InsR $(\mathrm{P}<0.05$ for DMix; $\mathrm{P}<0.01$ for metformin) protein expression were significantly higher in the DMix and metformin groups compared with the diabetic
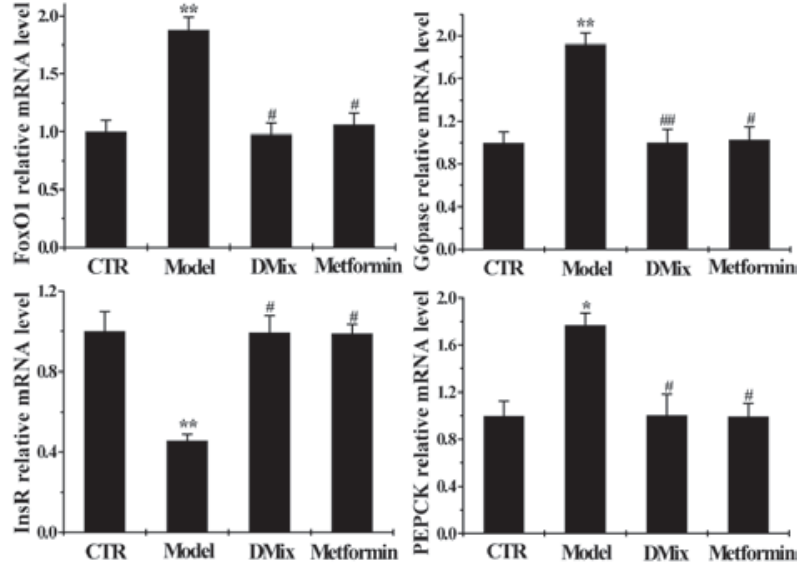

Figure 5. Expression levels of InsR, FoxO1, PEPCK and G6Pase mRNA as determined by reverse transcription-quantitative polymerase chain reaction. GAPDH was used as the internal control. All results are expressed as the mean \pm standard deviation of three independent experiments. ${ }^{*} \mathrm{P}<0.05$ and ${ }^{* *} \mathrm{P}<0.01$ vs. CTR group; ${ }^{\text {}} \mathrm{P}<0.05,{ }^{\# \#} \mathrm{P}<0.01$ vs. diabetic model group. CTR, control; InsR, insulin receptor $\beta$; FoxO1, forkhead box protein O1; PEPCK, phosphoenolpyruvate carboxykinase; G6Pase, glucose 6-phosphatase.

model group, whereas the FoxO1 $(\mathrm{P}<0.01)$, PEPCK $(\mathrm{P}<0.05)$ and G6Pase $(\mathrm{P}<0.05$ for DMix; $\mathrm{P}<0.01$ for metformin) levels were significantly lower.

\section{Discussion}

Diabetes may cause acute and chronic complications, which makes it a public health issue that requires global attention (20). The establishment of appropriate diabetic animal models is important for studying the pathogenesis of diabetes. STZ destroys islet $\beta$ cells and a low dose of STZ combined with a high-fat diet is the most common way to induce diabetes in rodents $(21,22)$. A previous in vivo study by the authors demonstrated that rats fed 6 weeks of a high-fat/high-sugar diet combined with two intraperitoneal injections of low-dose STZ, effectively established a stable and affordable rat model of diabetes (23). The same method was used in the present study to establish the Wistar rat model of diabetes.

Based on its clinical manifestations and features, diabetes is categorized as a dispersion-thirst disease in TCM (24). The incidence of diabetes is multifactorial and may be associated with genetic (25), environmental (26), dietary (27), lifestyle (27) factors. In TCM, type II diabetes may be treated with the Nourishing-'yin', Tonifying-'qi' method (28) or the Nourishing-'yin', Tonifying-qi, activating-blood (promoting blood flow) methods (29).

The DMix was prepared according to TCM theory and consisted of dendrobium, astragalus, salvia, rehmannia, pueraria, schisandra and earthworms. TCM suggests that this combination should promote body fluid production, eliminate dryness-heat and promote systemic blood flow, therefore, DMix may effectively prevent and treat type II diabetes and its complications $(15,16)$

A previous study by the authors revealed that 1,339 of the 41,023 liver genes examined were expressed differently in a rat model of diabetes compared with healthy rats (24). However, 
A
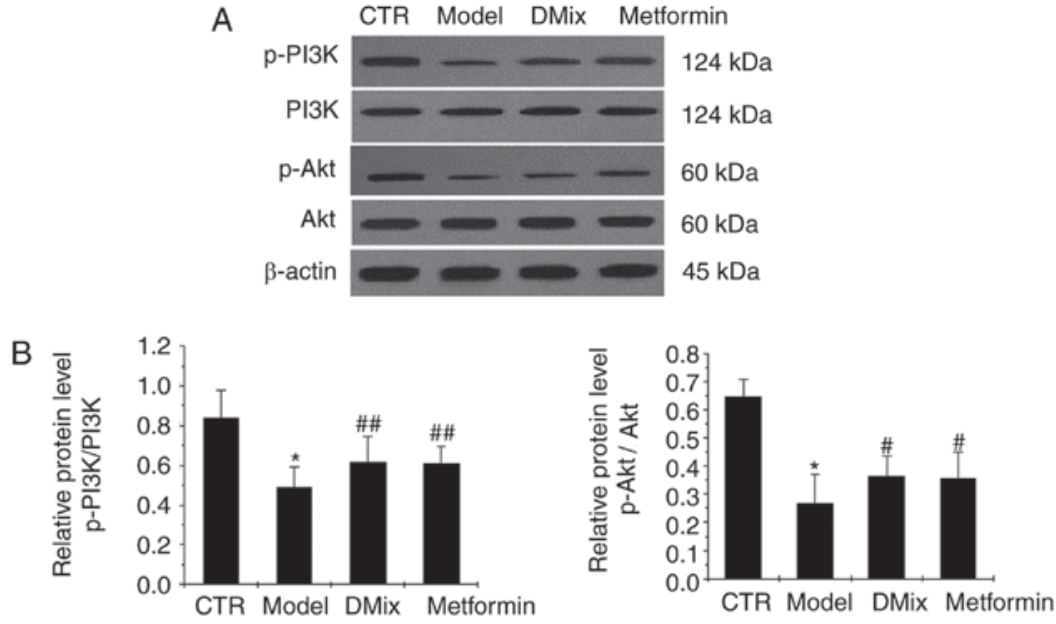

C

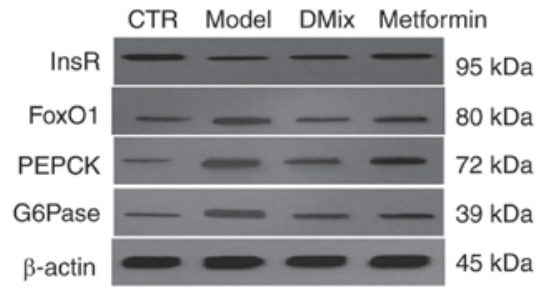

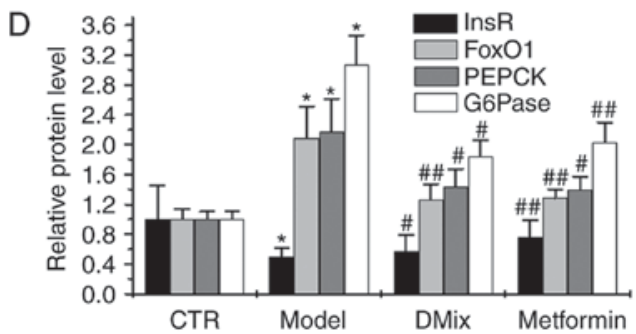

Figure 6. Protein expression levels of PI3K, p-PI3K, Akt, p-Akt, InsR, FoxO1, PEPCK and G6Pase as measured by western blot analysis. (A) Western blot analysis of the proteins. The western blot was quantified and the (B) p-PI3K/PI3K and p-Akt/Akt results are presented as bar charts. (C) Western blot analysis of InsR, FoxO1, PEPCK and G6Pase. (D) The western blot was quantified and presented as a bar chart; the band intensity was normalized to $\beta$-actin. "P<0.01 vs. CTR group; ${ }^{\#} \mathrm{P}<0.05$ and ${ }^{\# \#} \mathrm{P}<0.01$ vs. Model group. CTR, control; PI3K, phosphoinositide-3-kinase; Akt, protein kinase B; InsR, insulin receptor $\beta$; FoxO1, forkhead box protein O1; PEPCK, phosphoenolpyruvate carboxykinase; G6Pase, glucose 6-phosphatase; p-, phosphorylated.

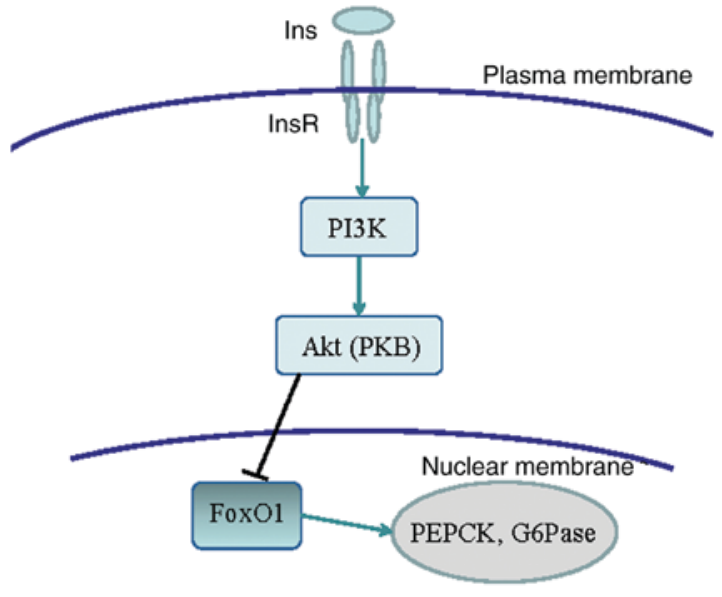

Figure 7. DMix regulates hepatic gluconeogenesis in diabetic rats via the PI3K/Akt signaling pathway. Ins, insulin; InsR, insulin receptor $\beta$; PI3K, phosphoinositide-3-kinase; Akt, protein kinase B; FoxO1, forkhead box protein O1; PEPCK, phosphoenolpyruvate carboxykinase; G6Pase, glucose 6-phosphatase.

only 380 genes were expressed differently in diabetic rats treated with DMix compared with healthy rats. This indicates that the healthy expression of $\sim 1,000$ genes was restored by DMix treatment (24). The previous study also suggested that DMix affected gene transcriptomes in vivo supporting the use of DMix as a potential treatment of Type II diabetes. Metformin, used here as a representative biguanide is an oral antidiabetic drug that lowers insulin resistance in vivo, improves hyperinsulinemia, minimizes liver gluconeogenesis and promotes the uptake and utilization of glucose in peripheral insulin-sensitive tissues (17). These effects lead to a reduction in blood glucose levels (30). Metformin was used in the present study as a positive control.

Animals in the diabetic model group had a significantly elevated FBG compared with the healthy control rats, but treatment with DMix or metformin significantly reduced FBG in the diabetic rats compared with the diabetic model rats at 3 , 6,9 and 12 weeks after treatment. The FBG levels were significantly lower in the metformin group at 3 and 6 weeks compared with the DMix group, but no significant differences between the two groups were observed at 9 and 12 weeks. This suggests that metformin had a faster hypoglycemic effect, whereas the hypoglycemic effect of DMix was more stable. GSP, a sensitive indicator of recent blood glucose levels is not affected by the current blood glucose concentration and instead reflects blood glucose levels over the previous 2-3 weeks. It was revealed that GSP was significantly elevated in the diabetic model group compared with the healthy control group. In addition, GSP levels were significantly lower in rats treated with DMix or metformin, which suggests that the hypoglycemic effect of DMix was similar to metformin.

The results indicated that Tch, TG and LDL levels were significantly elevated in the diabetic model group compared with the healthy control group. However, the levels of Tch, TG and LDL were significantly reduced following treatment with DMix compared with the model group. However only the 
levels of Tch and LDL were significantly reduced following treatment with metformin. A previous study demonstrated that in addition to reducing blood sugar levels, metformin significantly reduced cholesterol and LDL-C levels (31), which is consistent with the findings of the present study. Clinical complications of diabetes may involve multiple systems and organs, including the liver (32). A previous study revealed that the incidence of liver disease is $21-78 \%$ in type I and II diabetes and the risk of suffering mortality due to liver disease is higher for patients with type II diabetes compared with the general population (33). T2DM promotes the progression of NAFLD to NASH, liver fibrosis and liver cancer (34). In addition, it increases mortality rate associated with the liver (34). The correlation between high alanine ALT levels and reduced hepatic insulin sensitivity may be used to predict the development of type II diabetes (35). In the present study, ALT and AST levels were significantly elevated in the model group compared with the control group. Examination of the liver histopathology of diabetic rats revealed changes in the shape of the hepatocytes, a loss of the cord-like arrangement in the liver lobule, an enlarged hepatic sinus and an abnormal structure in the central vein and portal area. This suggests that liver damage in diabetic rats may be associated with inflammatory damage caused by dysregulated glucose and lipid metabolism, and by prolonged stimulation with high glucose. The ALT and AST levels in diabetic rats treated with DMix or metformin were significantly reduced 12 weeks after treatment compared with the diabetic model group. Liver histopathological examinations in diabetic rats treated with DMix or metformin revealed neatly arranged hepatocytes and improved hepatic cord and hepatic sinus structures. These observations suggest that DMix reduced elevated transaminase levels, improved liver histopathology and exerted a protective function on the liver. This is associated with the improvement of glycolipid metabolism in diabetic rats by DMix and the reduction of liver damage by hyperglycemia and hyperlipidemia.

A previous study demonstrated that hyperinsulinemia is an initiator of insulin resistance (36). Sustained high insulin levels may reduce the expression of insulin receptors in peripheral tissues reducing intracellular glucose utilization and further increasing insulin secretion via feedback stimulation (37). In the present study it was observed that insulin levels and insulin resistance were significantly increased in diabetic model rats compared with the healthy control rats, suggesting that hyperinsulinemia and insulin resistance had successfully been induced in the model rats. Compared with the diabetic model rats, the insulin levels and the insulin resistance index were significantly lower in the diabetic rats treated with DMix. This suggests that DMix treatment significantly alleviated hyperinsulinemia and significantly reduced insulin resistance.

The binding of insulin to InsR activates the PI3K/Akt signaling pathway and mediates insulin metabolism in peripheral tissues, including the liver and skeletal muscle (38). InsR is responsible for initiating intracellular insulin transmembrane signal transduction (37). Abnormal InsR expression or phosphorylation affect normal insulin signal transduction and these abnormalities induce insulin resistance and glucose metabolism disorders (39). Akt is critical for metabolism and cell growth (5). PI3K (23) functions as a second messenger to activate downstream Akt (40). In the present study, it was revealed that the phosphorylation of InsR, PI3K and Akt was significantly lower in the livers of diabetic model rats compared with the healthy controls. This may be associated with reduced InsR expression, leading to decreased signal transduction. This suggests that high blood glucose and insulin levels inhibited Akt activity, consistent with the findings of Tomas et al (41). InsR, p-PI3K/PI3K and p-Akt/Akt levels were significantly upregulated in diabetic rats following DMix treatment compared with the diabetic model rats, suggesting that DMix may partly regulate PI3K/Akt signaling, improve glucose and lipid metabolism and reduce insulin resistance in a rat model of diabetes.

FoxO factors are involved in a range of biological processes, including cell proliferation, apoptosis and energy metabolism (42). The transcription factor FoxO1 promotes the gene expression of key gluconeogenesis enzymes by binding to specific promoters (43). PEPCK is a rate-limiting enzyme critical to the first step of gluconeogenesis, where it is pivotal in the conversion of oxaloacetate to phosphorylate pyruvate (44). PEPCK levels in the livers of PEPCK transgenic mice are $\sim 7$-fold higher than in normal mice, leading to a significant increase in gluconeogenesis (45). The enzyme G6Pase helps to catalyze the hydrolysis of 6-phosphate glucose into glucose (46), the final step of gluconeogenesis. Therefore, increased G6Pase activity directly affects glycogen output. The binding of insulin to InsR activates the PI3K/Akt signaling pathway, which inactivates the phosphorylation of the downstream signaling molecule FoxO1 and induces its degradation. This reduces the expression of PEPCK and G6Pase enzymes associated with gluconeogenesis, and inhibits gluconeogenesis. The signal pathway is shown in Fig. $7(11,12)$. In the present study, it was revealed that FoxO1, PEPCK and G6Pase protein expression was significantly upregulated in the livers of diabetic model rats compared with the healthy control rats. The authors hypothesized that the reduced activity of InsR, p-PI3K and p-Akt decreased FoxO1 degradation, subsequently increasing the expression of the gluconeogenic enzymes PEPCK and G6Pase and resulting in an increased hepatic glucose output in the diabetic rats. In the present study, DMix significantly decreased FoxO1, PEPCK and G6Pase protein expression levels in the diabetic rats, thereby suppressing gluconeogenesis and improving glucose metabolism.

The results indicated that DMix treatment significantly reduced the levels of blood glucose, blood lipid, GSP and insulin in diabetic rats. These effects may be caused by an increase in the gene and protein expression of InsR, p-PI3K and $\mathrm{p}-\mathrm{Akt}$ in the insulin signaling pathway, and a reduction in the gene and protein expression of FoxO1, PEPCK and G6Pase. The results suggest that DMix suppressed gluconeogenesis, improved insulin resistance and effectively treated and prevented diabetes by regulating the gene and protein expression of key molecules in the insulin signaling pathway. Insulin levels in the blood are regulated by insulin secretion and insulin clearance, and receptor-mediated endocytosis is considered the primary mechanism of insulin clearance (47). Therefore, in addition to suppressing insulin secretion, DMix may reduce hyperinsulinemia by promoting the degradation 
and endocytosis of insulin. However, further study is required to investigate this.

\section{Acknowledgements}

The authors would like to thank Professor Shi Hong for her professional instruction.

\section{Funding}

The present study was supported by The National Natural Science Foundation of China (grant no. 81503438), The Project of the Department of Health of Fujian Province (grant no. 2014-1-69) and The Key Project of the Fujian Education Department (grant no. JA15228).

\section{Availability of data and materials}

The datasets used and/or analyzed during the current study are available from the corresponding author on reasonable request.

\section{Authors' contributions}

$\mathrm{XL}$ and HS conceived and designed the present study. XL, YC, XW, JZ, WY and MW performed the experiments. XL wrote and HS reviewed the manuscript. All authors read and approved the final version of the manuscript.

\section{Ethics approval and consent to participate}

The present study was approved by the Ethics Committee of Fujian University of Traditional Chinese Medicine (Fuzhou, China).

\section{Consent for publication}

Not applicable.

\section{Competing interests}

The authors declare that they have no competing interests.

\section{References}

1. Xu Y, Wang L, He J, Bi Y, Li M, Wang T, Wang L, Jiang Y, Dai M, $\mathrm{Lu} \mathrm{J}$, et al: Prevalence and control of diabetes in chinese adults. JAMA 310: 948-959, 2013.

2. Boj SF, van Es JH, Huch M, Li VS, José A, Hatzis P, Mokry M, Haegebarth A, van den Born M, Chambon P, et al: Diabetes risk gene and Wnt effector Tcf712/TCF4 controls hepatic response to perinatal and adult metabolic demand. Cell 151: 1595-1607, 2012.

3. Gao J, Duan C and Li LJ: The pathogenesis mechanisms of type 2 diabetes mellitus. Medical Recapitulate 21: 3935-3938, 2015 (In Chinese).

4. Steinbrunn T, Stühmer T, Sayehli C, Chatterjee M, Einsele H and Bargou RC: Combined targeting of MEK/MAPK and PI3K/Akt signalling in multiple myeloma. Br J Haematol 159: 430-440, 2012.

5. Bence KK: Hepatic PTP1B deficiency: The promise of a treatment for metabolic syndrome. J Clin Metab Diabetes 1: 27-33, 2010.

6. Hojlund K: Metabolism and insulin signaling in common metabolic disorders and inherited insulin resistance. Dan Med J 61: B4890, 2014.

7. Yang X and Dongming Su D: Effects of HRD1 expression in hepatic gluconeogenesis. Jiangsu Med J 13: 908-911, 2017 (In Chinese).
8. Enes P, Panserat S, Kaushik S and Oliva-Teles A: Nutritional regulation of hepatic glucose metabolism in fish. Fish Physiol Biochem 35: 519-539, 2009.

9. Staehr P, Hother-Nielsen O and Beck-Nielsen H: The role of the liver in type 2diabetes. Rev Endocr Metab Disord 5: 105-110, 2004.

10. Houde VP, Brule S, Festuccia WT, Blanchard PG, Bellmann K, Deshaies Y and Marette A: Chronic rapamycin treatment causes glucose intolerance and hyperlipidemia by upregulating hepatic gluconeogenesis and impairing lipid deposition in adipose tissue. Diabetes 59: 1338-1348, 2010.

11. Gross DN, Wan M and Birnbaum MJ: The role of FoxO in the regulation of metabolism. Curr Diab Rep 9: 208-214, 2009.

12. Wang Q, Wang N, Dong M, Chen F, Li Z and Chen Y: GdC13 reduces Hyperglycaemia through Akt/FoxOl-induced suppression of hepatic gluconeogenesis in Type 2 diabetic mice. Clin Sci (Lond.) 127: 91-100, 2014

13. Tang WL, Wang YM, Du WM, Chen BY and Cheng NN: Analysis of adverse drug reaction of antidiabetic agents according to documents within forty-two years in China. Chin J New Drugs Clin Rem 21: 753-768, 2002 (In Chinese).

14. Zhu Y, Song C, Huo H, et al: Research of traditional chinese medicine in treating type diabetes and insulin resistance. World Chin Med 10: 135-137, 2015 (In Chinese).

15. Ke Z, Hou X and Jia X: Application idea and method discussion of Chinese materia medica with hypoglycemic effect based on active ingredients. Chin Traditional Herbal Drugs 47: 1797-1805, 2016 (In Chinese).

16. Shi H, Zhang X, Cui Yi, et al: Clinical sequential treatment and experimental studies of DC for diabetes mellitus. Clinical J Chin Med 5: 1-3, 2013 (In Chinese).

17. Xin J, Wang Q, Chen L, et al: Clinical sequential treatment and experimental studies on the effect of dendrobium powder on insulin resistance in type 2 diabetes mellitus. China Modern Doctor 48: 58-59, 2010 (In Chinese).

18. Liu Y and He R: Fasting induces a high level of 3-nitrotyrosine in the brain of rats. Neurosci Lett 472: 204-209, 2010.

19. Livak KJ and Schmittgen TD: Analysis of relative gene expression data using real-time quantitative PCR and the 2(-Delta Delta C(T)) method. Methods 25: 402-408, 2001.

20. Whiting DR, Guariguata L, Weil C and Shaw J: IDF diabetes atlas: Global estimates of the prevalence of diabetes for 2011 and 2030. Diabetes Res Clin Pract 94: 311-321, 2011.

21. Wang J, Shen X, Fu C, et al: Type 2 rat diabetic models induced by high-fat feeding combined with Streptozocin. Jilin Med J 32: 629-633, 2011 (In Chinese)

22. Matteucci E and Giampietro O: Proposal open for discussion: Defining agreed diagnostic Procedures in experimental diabetes research. J Ethnopharmacol 115: 163-172, 2008.

23. Lin X, Wang Q, Xin J, et al: The study of establishing a highly stable and successful diabetic rat model. Chin J Gerontology 5: 2051-2054, 2013 (In Chinese).

24. Bai X, Huang Y and Xu Y: The knowledge of modern physicians on diabetes pathogenesis of tcm. Guangming J Chin Med 30: 206-209, 2015 (In Chinese).

25. Xu Q, Liu Y, Cong YB, Zheng YY, Zhang JP and Shi H: Gene expression and microarray investigation of dendrobium mixture as progressive therapy for the treatment of type 2 diabetes mellitus. Tropical J Pharmaceutical Res 12: 195-201, 2013.

26. Patel DK, Kumar R, Laloo D and Hemalatha S: Diabetes mellitus: An overview on its pharmacological aspects and reported medicinal plants having antidiabetic activity. Asian Pac J Trop Biomed 2: 411-420, 2012 .

27. Li X, Li S, Huang T and Bai H Effect of aerobic exercise and diet intervention on male college students with variants of FTO gene. J Shanghai University Sport 38: 95-99, 2014 (In Chinese).

28. Zhang Y: Clinical study on Ziyin Yiqi pills in treatment of diabetic nephropathy. China J Chin Med 20: 1593-1594, 2015.

29. Yu WZ, Chen LJ and Shi H: Effect of the method of nourishing-'yin', Tonifying-'qi' and activating-blood on caspase-3 mrna expression in pancreas of aged diabetic rats. Lishizhen Medicine and Materia Medica Res 23: 2040-2042, 2012 (In Chinese).

30. Stumvoll M, Nurjhan N, Perriello G, Dailey G and Gerich JE: Metabolic effects of metformin in non-insulin-dependent diabetes mellitus. N Engl J Med 333: 550-554, 1995.

31. Wulffelé MG, Kooy A, de Zeeuw D, Stehouwer CD and Gansevoort RT: The effect of metformin on blood pressure, plasma cholesterol and triglycerides in type 2 diabetes mellitus: A systematic review. J Intern Med 256: 1-14, 2004. 
32. Nakahara T, Hyogo H, Yoneda M, Sumida Y, Eguchi Y, Fujii H, Ono M, Kawaguchi T, Imajo K, Aikata H, et al: Type 2 diabetes mellitus is associated with the fibrosis severity in patients with nonalcoholic fatty liver disease in a large retrospective cohort of Japanese patients. J Gastroenterol 49: 1477-1484, 2014.

33. Zhou YJ, Li YY, Nie YQ, Huang CM and Cao CY: Natural course of nonalcoholic fatty liver disease in southern China: A prospective cohort study. J Dig Dis 13: 153-160, 2012.

34. Bhaha N, Younes R and Bugianesi E: Epidemiology and natural history of patients with NAFLD. Curr Pharm Des 19: 5169-5176, 2013.

35. Vozarova B, Stefan N, Lindsay RS, Saremi A, Pratley RE, Bogardus C and Tataranni PA: High alanine aminotransferase is associated with decreased hepatic insulin sensitivity and predicts the development of type 2 diabetes. Diabetes 51: 1889-1895, 2002.

36. Nolan CJ, Ruderman NB, Kahn SE, Pedersen O and Prentki M; Response to Comments on Nolan: Insulin resistance as a physiological defense against metabolic stress: Implications for the management of subsets of type 2 diabetes. Diabetes 64: 673-686, 2015.

37. Gray SL, Donald C, Jetha A, Covey SD and Kieffer TJ: Hyperinsulinemia precedes insulin resistance in mice lacking pancreatic beta-cell leptin signaling. Endocrinology 151: 4178-1486, 2010.

38. Wang Y, Li B, Zhang W, Liu Y, Xue P, Ma J and Li Y: Impaired $\mathrm{PI} 3 \mathrm{~K}$ Akt expression in liver and skeletal muscle of ovariectomized rats. Endocrine 44: 659-665, 2013.

39. Wang YM, Lin XF, Shi CM, Lu L, Qin ZY, Zhu GZ, Cao XG, Ji CB, Qiu J and Guo XR: $\alpha$-Lipoic acid protects 3T3-L1, adipocytes from NYGGF4 (PID1) overexpression-induced insulin resistance through increasing phosphorylation of IRS-1 and Akt. J Bioenerg Biomembr 44: 357-363, 2012.

40. Han K, Xu X, Chen G, Zeng Y, Zhu J, Du X, Zhang Z, Cao B, Liu $Z$ and Mao X: Identification of a promising PI3K inhibitor for the treatment of multiple myeloma through the structural optimization. J Hematol Oncol 7: 9, 2014.
41. Tomas NM, Masur K, Piecha JC, Niggemann B and Zänker KS: Akt and phospholipase $C \gamma$ are involved in the regulation of growth and migration of MDA-MB-468 breast cancer and SW480 colon cancer cells when cultured with diabetogenic levels of glucose and insulin. BMC Res Notes 5: 214, 2012.

42. Ropelle ER, Pauli JR, Prada P, Cintra DE, Rocha GZ, Moraes JC, Frederico MJ, da Luz G, Pinho RA, Carvalheira JB, et al: Inhibition of hypothalamic Foxol expression reduced food intake in diet-induced obesity rats. J Physiol 587: 2341-2351, 2009.

43. Fabbrini E, Sullivan S and Klein S: Obesity and nonalcoholic fatty liver disease: Biochemical, metabolic, and clinical implications. Hepatology 51: 679-689, 2010.

44. Gómez-Valadés AG, Vidal-Alabró A, Molas M, Boada J, Bermúdez J, Bartrons R and Perales JC: Overcoming diabetes-induced hyperglycemia through inhibition of hepatic phosphoenolpyruvate carboxykinase (GTP) with RNAi. Mol Ther 13: 401-410, 2006.

45. Kim YK, Lee GS, Jung EM, Hyun SH, Hwang WS and Jeung EB: Generation of fibroblasts overexpressing liver-specific PEPCK in a miniature pig model of human type 2 diabetes mellitus. Mol Med Rep 6: 45-50, 2012.

46. Hutton JC and O'Brien RM: Glucose-6-phosphatase catalytic subunit gene family. J Biol Chem 284: 29241-29245, 2009.

47. Costa-Júnior JM, Ferreira SM, Protzek AO, Santos GJ, Cappelli AP, Silveira LR, Zoppi C, de Oliveira CA, Boschero AC, Carneiro EM and Rezende LF: Endurance training inhibits insulin clearance and IDE expression in Swiss mice. PLoS One 10: e0118809, 2015

This work is licensed under a Creative Commons Attribution-NonCommercial-NoDerivatives 4.0 International (CC BY-NC-ND 4.0) License. 